\title{
Initial Rotor Position Detection in PMSM based on Low Frequency Harmonic Current Injection
}

\author{
Duro Basic ${ }^{*}$, Francois Malrait ${ }^{*}$ and Pierre Rouchon ${ }^{\dagger}$ \\ ${ }^{*}$ Duro Basic and Francois Malrait are with Schneider Toshiba Inverter, rue Andre Blanchet, 27120 Pacy sur Eure, \\ France, e-mail:duro.basic@schneider-electric.com,francois.malrait@schneider-electric.com
}

${ }^{\dagger}$ Pierre Rouchon is with Mines-ParisTech, Centre Automtique et Systèmes, Mathématiques et Systémes, 60 Bd. SaintMichel, 75272 Paris Cedex 06, France, e-mail: pierre.rouchon@mines-paristech.fr

\begin{abstract}
This paper presents an approach for the initial rotor position detection in permanent magnet synchronous machine (PMSM) drives. It is based on injection of low frequency current and excitation of small rotor speed oscillations. In this way information about spatial position of the rotor flux can be retrieved from the stator voltage response regardless of the rotor (average) speed. The method presented is based on the standard synchronous reference frame proportional-integral (SRF PI) current controller augmented by a bank of harmonic integral (I) controllers. Such expanded current controller typically used in selective active filters provides simultaneously precise control of the harmonic current injection and spectral decomposition of the applied stator voltage. In the theoretical analyses presented relationships between the rotor flux position information and outputs of the harmonic I controllers are established. Based on this analysis a simple rotor position tracking and magnet flux polarity detection system is constructed. Experimental results presented demonstrate feasibility of this approach for initial rotor position detection in PMSM drives.
\end{abstract}

Keywords - Variable speed drive, Sensorless control, Ac machine, Permanent magnet motor.

\section{INTRODUCTION}

For effective torque control in PMSM by variable speed drives, the drive control system requires information about spatial position of the rotor flux vector in the machine. The rotor flux vector position can be measured (PMSM) or estimated (induction machines) if the rotor position is measured by using a rotor speed or position sensor (typically an absolute or incremental encoder). The rotor flux position can be also estimated without using the rotor speed/position sensor. This is a preferable option as the sensor is relatively expensive and mechanically and electrically sensitive so the drive system is cheaper and more reliable without it. In this case the rotor flux position is estimated from the machine model and stator voltage equation. Due to the model parametric and delivered stator voltage errors this approach is effective when the stator frequency and consequently the internal emf. (electromotive force) induced by the flux are sufficiently high so that flux position can be reliably detected from the stator voltages. This is the case at sator frequencies above typically $5-10 \%$ of the nominal frequency. Such control mode is known as the fundamental frequency based (position) sensorless control.
For tracking of the rotor flux at low stator frequencies down to zero speed numerous techniques based on an auxiliary signal injection have been proposed [1-13] as an alternative or for enhancement of the fundamental frequency based sensorless control at low speeds. Normally injections of a voltage or current signal into the stator winding at a frequency different from the fundamental frequency are used to retrieve the rotor flux position from the stator current or voltage response. Currently, in standard variable speed drives, application of the harmonic injection is mostly limited for starting of PMSM to initialize the control system with correct initial rotor position (it minimizes uncontrolled rotor movements when the fundamental frequency sensorless control is launched). Also, in cases when the PMSM is equipped with an incremental encoder, absolute rotor (flux) position needs to be detected prior start ups. Thus it is of great interest to validate various methods for effective initial rotor magnet position detection in standstill. Ideally such methods should produce minimal rotor movements.

The harmonic injection methods can be classified in two major groups: the high [1-7] and low frequency [8-13] harmonic injection methods. The high frequency injection methods apply relatively high frequency (typically $0.5 \mathrm{~Hz}-$ $2 \mathrm{kHz}$ ) periodic harmonic injection in form a rotational or pulsating vector. The injected signal is used to estimate rotor position dependant effects on the stator inductance matrix which variation is caused by either rotor geometric saliency [4,5] or magnetic saturation [1-3]. The rotor position is tracked via detection of spatial position of the stator winding inductance saliency. Tracking of the inductance saliency can give information only about spatial position of the rotor/saliency axis. The magnet flux polarity is normally detected $(0-\pi$ ambiguity resolution) by exploiting magnetic saturation effects. This procedure is normally needed only on initial start-ups as the rotor tracking error is maintained small during the run state. In some solutions the same high frequency based rotor position tracking is used in the start-up phase as well in run state (as enhancement of low speed performance of fundamental frequency based sensorless algorithms). In this case the magnetic polarity (initial rotor flux position) is tested only on start-ups after the rotor axis position tracking has converged. Usually voltage pulses are applied along the $\mathrm{d}$ axis in both directions for the polarity test [3]. The magnet flux polarity is oriented in the controller $\mathrm{d}$ axis direction in which the test voltage pulse produces higher current peak. The magnet polarity can be tracked continuously with the tracking of the saliency axis [1]. In this approach, polarity/phase of the second harmonic in 
the voltage/current response of the machine is continuously observed as it is linked with the saturation $\left(2^{\text {nd }}\right.$ harmonic implies half wave asymmetry which is caused by saturation) [4]. In this case the initial rotor position ambiguity is automatically corrected by 0 or $\pi$ when the saliency axis tracking converged so there is no need for special magnet polarity test (faster start-up [1]). For completeness we have to mention so called single point detection methods which detect rotor position in regular intervals (no continuous rotor position tracking) by applying signal injection within a short test sequence [6]. For the initial rotor position detection only, the test sequence can utilise large signals injected from various spatial directions for simultaneous detection of the rotor flux position and polarity [7]. Such methods are effective even if the saliency may not be effectively detected by the small signal injection.

For the rotor flux position detection in ac machines so called low frequency harmonic current vector injection can be exploited [8-13]. In this context, the term 'low frequency' means that the injected harmonic frequency falls within the mechanical bandwidth of the mechanical subsystem. For the $50 \mathrm{~Hz}-60 \mathrm{~Hz}$ machines typical injection frequency would be in the $25 \mathrm{~Hz}-50 \mathrm{~Hz}$ frequency range. The goal of such harmonic current injection is to introduce small torque oscillations in order to excite small rotor speed oscillations capable of inducing detectable voltage oscillations in the stator winding. From the phase information of the detected voltage oscillations it is possible to deduce information about the rotor position. The low frequency injection has been already proposed for initial rotor position detection in PMSM [8]. It can not detect the magnet flux polarity and thus similar techniques to that used in conjunction with the high frequency based methods can be used for the magnet polarity detection. The low frequency injection technique has been employed also for the sensorless control (continuous tracking of the rotor flux position) of induction machines $[9,10]$ and PMSMs [11-13]. However it is found that the control dynamics is inferior to that achievable with the fundamental frequency [12] or high frequency [17] based sensorless control methods. Typically an alternating harmonic current vector is injected from the controller reference frame as it minimizes the torque oscillations once controller is aligned with the rotor axis $[8,9]$. One implementation approach for this technique is to use the use standard fundamental current SRF PI controller with sufficient bandwidth (capable of controlling the harmonic injection) and stator voltage equation to retrieve the internal induced emf. [9,10]. However when using such approach knowledge of several machine parameters are required and additional demodulation processing to retrieve the rotor position error signal is needed. It makes the overall rotor position estimation algorithm complex and motor parameter dependant. Also the harmonic current injection control is not precise and a steady state error in the injection current can be created.

In this paper we present an approach for the initial rotor (flux) position detection method in PMSM based on the low frequency current injection. This approach differs from the previous work in the manner how the controlled harmonic current injection is performed and relevant stator voltage spectral component is retrieved. We use a current controller suitable for simultaneous harmonic current injection and retrieval of voltage spectral component to be used as an error signal in the rotor flux position tracking. The current control is augmented by two sets of SRF harmonic I controllers rotating with respect to the fundamental frequency control frame at the harmonic frequency $\Omega$ in the positive and negative directions (positive and negative sequence harmonic controller). Due to harmonic integrators centered at $\pm \Omega$ (seen in the fundamental frequency SRF) the current controller is capable of enforcing current injection at both harmonic sequences with zero steady-state tracking errors. Simultaneously (due to infinite gain of integrators at frequency of respective SRF), the current integrator outputs (including standard fundamental frequency integral controllers) directly separate voltage space vector spectral components of the stator voltage without need for any additional signal processing and without requiring any motor parameter. Similar current controllers based on banks of multiple reference frame harmonic controllers [14] or their stationary frame equivalents (resonant filters) [15] can be found in active power filters when selective compensation of targeted harmonics is needed.

The paper is organized as follows. Initially in Section II we present a salient pole PMSM model written in an arbitrary reference frame in a very compact complex form. Further, assuming a stiff harmonic current injection, we in Section III derive in systematic manner relations between the harmonic I controller outputs and rotor flux position error by using averaging techniques and perturbation theory [16]. Two special cases with rotational (Section IV) and pulsating (Section V) harmonic injection are considered in more detail. The magnet flux polarity detection and current controller structure are discussed in Sections VI and VII. Finally in Section VIII we introduce a simple rotor flux position tracking loop and in Section IX we present some preliminary experimental results. These experimental results indicate that the initial rotor flux position detection in PMSM based on the proposed current control strategy and low frequency injection is practically feasible.

\section{PMSM MODEL}

We will start from the standard salient pole PMSM model written in the controller reference frame rotating at arbitrary stator frequency $\omega_{\mathrm{s}}$. In general case the controller reference frame without position sensor may not be aligned with the flux/rotor frame. This situation is illustrated in Fig. 1 where the rotor frame is advanced by an angle $\varepsilon$ with respect to the controller frame ( $\varepsilon$ represents alignment error between the rotor and controller frames).

$$
\begin{aligned}
& v_{s}=R_{S} i_{s}+L \frac{d i_{s}}{d t}+\Delta L \frac{d i_{s}{ }^{*}}{d t} e^{j 2 \varepsilon}+ \\
& +j \omega_{s}\left(L_{i_{S}}-\Delta L i_{s}^{*} e^{j 2 \varepsilon}\right)+j \omega\left(2 \Delta L_{i_{S}}{ }^{*} e^{j 2 \varepsilon}+\bar{\varphi}_{m} e^{j \varepsilon}\right) \\
& \varphi_{S}=L_{i_{S}}+\Delta L_{i_{S}}{ }^{*} e^{j 2 \varepsilon}+\bar{\varphi}_{m} e^{j \varepsilon} \\
& L=\frac{L_{d}+L_{q}}{2} \\
& \Delta L=\frac{L_{d}-L_{q}}{2}
\end{aligned}
$$




$$
\begin{aligned}
& \tau_{e}=\frac{3}{2} n_{p} \operatorname{Im}\left(\varphi_{s}{ }^{*} i_{S}\right) \\
& \frac{d \omega}{d t}=\frac{n_{p}}{J}\left(\tau_{e}-\tau_{l}\right)
\end{aligned}
$$

The stator current and voltage variables are complex ('*', means conjugation), and 'ored' variables denote steady state/average variables. The term $\bar{\varphi}_{m}$ represents the rotor magnet flux. The inductance terms $\mathrm{L}$ and $\Delta L$ are the average and saliency inductance respectively. Non negligible inductance saliency typically exists in PMSMs with rotor geometric saliency.

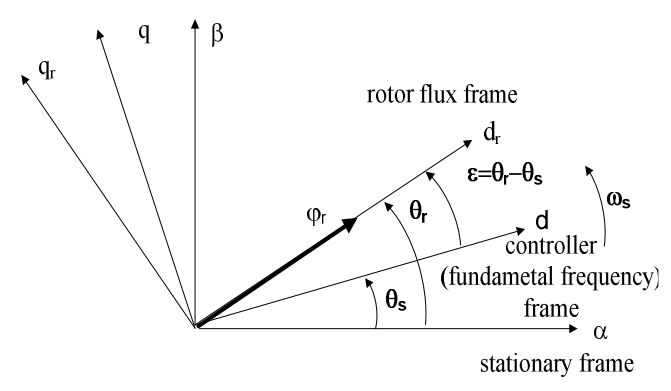

Fig. 1. Rotor flux and fundamental frequency current controller reference frame positions with position error $\varepsilon$.

\section{PERTURBATION ANLYSIS}

We will start the analysis assuming that the stator current vector is perturbed by and arbitrary elliptic harmonic signal injection:

$$
i_{s}=\bar{i}_{s}+\delta i_{s} \quad \delta i_{s}=I^{+} e^{j \Omega t}+I^{-} e^{-j \Omega t}
$$

Small signal perturbations around the steady state point are designed by a prefix $\delta$. During start ups, the average stator current is assumed to be zero.

Due to the injected perturbation current, the PMSM machine torque will have the following average and oscillating torques and speed components (calculations based on averaging and perturbation techniques) [16]:

$$
\begin{gathered}
\dot{\bar{\omega}}=\frac{3 n_{p}{ }^{2}}{2 J} \operatorname{Im}\left(2 \Delta L I^{+} I^{-} e^{-j 2 \varepsilon}\right) \\
\delta \dot{\omega}=\frac{3 n_{p}^{2}}{2 J} \operatorname{Im}\left(\begin{array}{l}
\bar{\varphi}_{m} I^{+} e^{-j \varepsilon} e^{j \Omega t}+\Delta L I^{+^{2}} e^{-j 2 \varepsilon} e^{j 2 \Omega t}+ \\
+\bar{\varphi}_{m} I^{-} e^{-j \varepsilon} e^{-j \Omega t}+\Delta L I^{-2} e^{-j 2 \varepsilon} e^{-j 2 \Omega t}
\end{array}\right) \\
\delta \omega=-\frac{3 n_{p}{ }^{2}}{2 J} \operatorname{Re}\left(\begin{array}{l}
\frac{\bar{\varphi}_{m} I^{+} e^{-j \varepsilon}}{\Omega} e^{j \Omega t}+\frac{\Delta L I^{+^{2}} e^{-j 2 \varepsilon}}{2 \Omega} e^{j 2 \Omega t}- \\
-\frac{\bar{\varphi}_{m} I^{-} e^{-j \varepsilon}}{\Omega} e^{-j \Omega t}-\frac{\Delta L I^{-2} e^{-j 2 \varepsilon}}{2 \Omega} e^{-j 2 \Omega t}
\end{array}\right)
\end{gathered}
$$

In these derivations we have assumed that the load torque is a static function of the rotor speed. In this case effect of the load torque perturbation caused by the speed perturbation can be neglected (according the averaging technique [16]). A simplified physical explanation is that the speed perturbation excited by the motor torque perturbation will be proportional to $1 / \Omega$ (see
(10)). Consequently in integration of (6), effect of the load torque perturbation (if taken into account) could be neglected as it would result in the load torque related speed perturbation proportional to $1 / \Omega^{2}$. For resonant loads where the load torque is a dynamic function of the speed, above assumption is valid only if the harmonic frequency is not in vicinity of such resonance frequencies.

After introducing (7) and (10) into (1) we can identify multiple spectral components (at different frequencies and sequences) in the stator voltage response which are excited by the harmonic current injection and related torque and rotor speed oscillations. The stator voltage response in (11) is composed of at the fundamental frequency and the first and second harmonic components of the injection frequency (with positive and negative sequence):

$$
v_{S}=V^{0}+V^{+} e^{j \Omega t}+V^{-} e^{-j \Omega t}+U^{+} e^{+j 2 \Omega t}+U^{-} e^{-j 2 \Omega t}
$$

Of particular interest for the rotor flux position detection are two stator voltage vector components $V^{+}$ and $V^{-}$rotating at the injection frequency $\pm \Omega$ but in opposite directions. After (7), (10) are inserted into (1) we can find theoretical expressions for these components:

$$
\begin{aligned}
& V^{+}=R_{S} I^{+}+j \Omega L I^{+}+j \Omega \Delta L I^{-} e^{j 2 \varepsilon}+j 2 \bar{\omega} \Delta L I^{-} e^{j 2 \varepsilon}- \\
& -j \frac{3 n_{p}^{2}}{4 J \Omega}\left(\Delta L^{2}\left(I^{+^{2}}-I^{-2} e^{j 4 \varepsilon}\right) I^{+}+\bar{\varphi}_{m}{ }^{2}\left(I^{+}-I^{-} e^{j 2 \varepsilon}\right)\right) \\
& V^{-}=R_{S} I^{-}-j \Omega L I^{-}-j \Omega \Delta L I^{+} e^{j 2 \varepsilon}+j 2 \bar{\omega} \Delta L I^{+} e^{j 2 \varepsilon}- \\
& -j \frac{3 n_{p}{ }^{2}}{4 J \Omega}\left(\Delta L^{2}\left(I^{+^{2}} e^{j 4 \varepsilon}-I^{-2}\right) I^{-}+\bar{\varphi}_{m}{ }^{2}\left(I^{+} e^{j 2 \varepsilon}-I^{-}\right)\right)
\end{aligned}
$$

From (12) and (13) we can conclude that these voltage components are functions of the rotor flux angular position error $\varepsilon$. Thus they can be used for tracking of the rotor position. We will further consider two typical types of the harmonic injections: rotational and alternating current vector injections.

\section{ROTATIONAL HARMONIC CURRENT INJECTION}

In this special case a rotational harmonic current vector is applied. Thus we have the following perturbation current vector:

$$
\delta_{i_{S}}=I e^{j \Omega t} \text {, i.e. } I^{+}=I, I^{-}=0
$$

We assume only the positive sequence harmonic injection, while the negative sequence harmonic current vector is regulated to zero. In this case the rotor flux position error is conveyed in the negative sequence voltage response at the harmonic frequency:

$$
V^{-}=-j\left(\Delta L(\Omega-2 \bar{\omega})+\frac{3 n_{p}{ }^{2}}{4 J \Omega} \bar{\varphi}_{m}{ }^{2}\right) I e^{j 2 \varepsilon}
$$

In fact the real part ( $\mathrm{d}$ axis component) of the negative sequence voltage vector component (retrieved from the negative sequence I controller output In Fig. 2) is sine function of 2 times the rotor position error:

$$
V_{d}^{-}=\operatorname{Re}\left(V^{-}\right)=\left(\Delta L(\Omega-2 \bar{\omega})+\frac{3 n_{p}^{2}}{4 J \Omega} \bar{\varphi}_{m}^{2}\right) I \sin (2 \varepsilon)
$$


It is important to notice that this rotor flux position error signal will exist in all PMSM machines, even if the inductance saliency term is zero $(\Delta L=0)$. In fact the saliency term appears as a disturbance factor which introduces magnitude dependence on the harmonic injection frequency and average rotor speed. This is in sharp contrast with various high frequency injection methods which exploit the inductance saliency for the rotor position tracking.

With this type of injection there will be no average component in the motor torque caused by the harmonic excitation $\left(\bar{\tau}_{e}=0\right)$ in presence of the inductance saliency. However perpetual speed/torque oscillations will be unfavourably present even if the control system is perfectly aligned with the rotor reference frame:

$$
\delta \omega=-\frac{3 n_{p}^{2}}{2 J \Omega}\left(\frac{\Delta L I^{2}}{2} \cos (2 \Omega t)+\bar{\varphi}_{m} I \cos (\Omega t)\right)
$$

This is major disadvantage of the rotational harmonic injection which makes it less suitable for practical applications.

\section{Pulsating Harmonic CurRent Injection}

We can apply two harmonic current vectors, with same magnitudes and rotating in opposite directions at the harmonic frequency $\Omega$ :

$$
I^{-}=I^{+}=\frac{I}{2} \quad \delta i_{s}=I \frac{e^{j \Omega t}+e^{-j \Omega t}}{2}=I \cos (\Omega t)
$$

This case is equivalent to applying a pulsating (alternating) harmonic current vector aligned with the controller $\mathrm{d}$ axis. In this case, to retrieve the rotor position error, we will combine both sequences of the stator voltage component at the injection frequency $(V)$. The $\mathrm{d}$ axis component of the combined voltage $V$ is dependant on the sine of two times the rotor flux position error:

$$
\begin{aligned}
& V=V^{-}-V^{+}=V_{d}+j V_{q} \\
& V_{d}=\operatorname{Re}(V)=\left(\Omega \Delta L+\frac{3 n_{p}{ }^{2}}{4 J \Omega}|\bar{\varphi}|_{m}{ }^{2}\right) I \sin (2 \varepsilon)+ \\
& +\frac{3 n_{p}{ }^{2}}{16 J \Omega} \Delta L^{2} I^{3} \sin (4 \varepsilon)
\end{aligned}
$$

With this type of excitation an average torque component can be produced if the machine axis inductance are not same (saliency exists). Thus some rotor movements can be produced with this type of excitation if the machine is lightly loaded.

$$
\bar{\tau}_{e}=\frac{3}{4} n_{p} \Delta L I^{2} \sin (2 \varepsilon)
$$

However an important advantage of the pulsating signal injection is that there is no speed and torque oscillations when the rotor flux and controller reference frames are well aligned (perturbation current applied in the flux direction). Thus for practical applications this type of harmonic injection is preferable.

\section{Magnet FluX Polaritity Dectection}

From the analysis presented we can see that the magnet flux position error signal is a function of double rotor flux position angular error $(2 \varepsilon)$. This fact means that in PMSM machines it is possible to detect only direction of the rotor flux axis but not its orientation or flux polarity $(0-\pi$ ambiguity). However if there is inductance saliency in the PMSM machine it is possible to detect the magnet flux polarity by using an extension of the proposed current controller. By using two sets of additional harmonic I controllers, placed in the frames rotating in opposite directions at 2 times injection frequency $( \pm 2 \Omega)$, it is possible to isolate the second harmonic voltage components $\left(\mathrm{U}^{+}\right.$and $\left.\mathrm{U}^{-}\right)$in (11). It is possible to show that with rotational injection, these voltages are:

$$
\begin{aligned}
U^{+} & =-j \frac{3 n_{p}^{2}}{2 J \Omega} \bar{\varphi}_{m} \frac{\Delta L I^{2}}{4} e^{-j \varepsilon} \\
U^{-} & =-j \frac{3 n_{p}^{2}}{2 J \Omega} \frac{5}{4} \Delta L I^{2} \bar{\varphi}_{m} e^{j 3 \varepsilon}
\end{aligned}
$$

Of particular interest is the $\mathrm{U}^{+}$(positive sequence $2^{\text {nd }}$ harmonic) component which is function of the angular error $\varepsilon$ only. The flux polarity can be unambiguously detected by analyzing sign of $\mathrm{R}_{\mathrm{e}}\left(\mathrm{U}^{+}\right)=\mathrm{U}_{\mathrm{d}}^{+}$:

$$
U_{d}^{+}=\operatorname{Re}\left(U^{+}\right)=\frac{3 n_{p}^{2}}{2 J \Omega} \bar{\varphi}_{m} \frac{\Delta L I^{2}}{4} \sin \varepsilon
$$

In the case of the alternating injection, the magnet polarity can be determined from the composite signal $\mathrm{U}$ :

$$
U_{d}=R_{e}\left(U^{+}-U^{-}\right)
$$

If the PMSM machine saliency $\Delta \mathrm{L}$ is zero, the magnet flux polarity detection can be resolved by using the iron core saturation effects only. For example, in case of the alternating signal injection, an asymmetry between the positive and negative half periods of the injected voltage will result again in the second harmonic which is retrieved by the second harmonic I controllers.

\section{SELECTIVE CURRENT CONTROLLER}

The controlled harmonic current injected is performed by the current controller shown in Fig. 2. The control is consisted, in addition to the standard fundamental frequency SRF PI controller with two sets of the harmonic current integral controllers which gain is $\mathrm{K}_{\mathrm{ih}}$. These controllers are placed in respective SRFs rotating at the injection frequency $\Omega$ in the both directions (sequences). With these controllers the harmonic current injection is performed with zero steady sate error. For detection of the second voltage harmonic at $2 \Omega$ (for magnet polarity tracking) two additional sets of harmonic I controllers are added (rotating in respective SRF at $\pm 2 \Omega$ ).

The stator voltage response to the perturbation current/speed perturbation is reflected at the outputs of the integral controllers and is split into dominant spectral components $(\mathrm{d}, \mathrm{q})$ and sequences $(+,-)$ due to infinite gains of the I controller cantered at $\omega_{\mathrm{s}}, \pm \Omega$ and $\pm 2 \Omega$. 


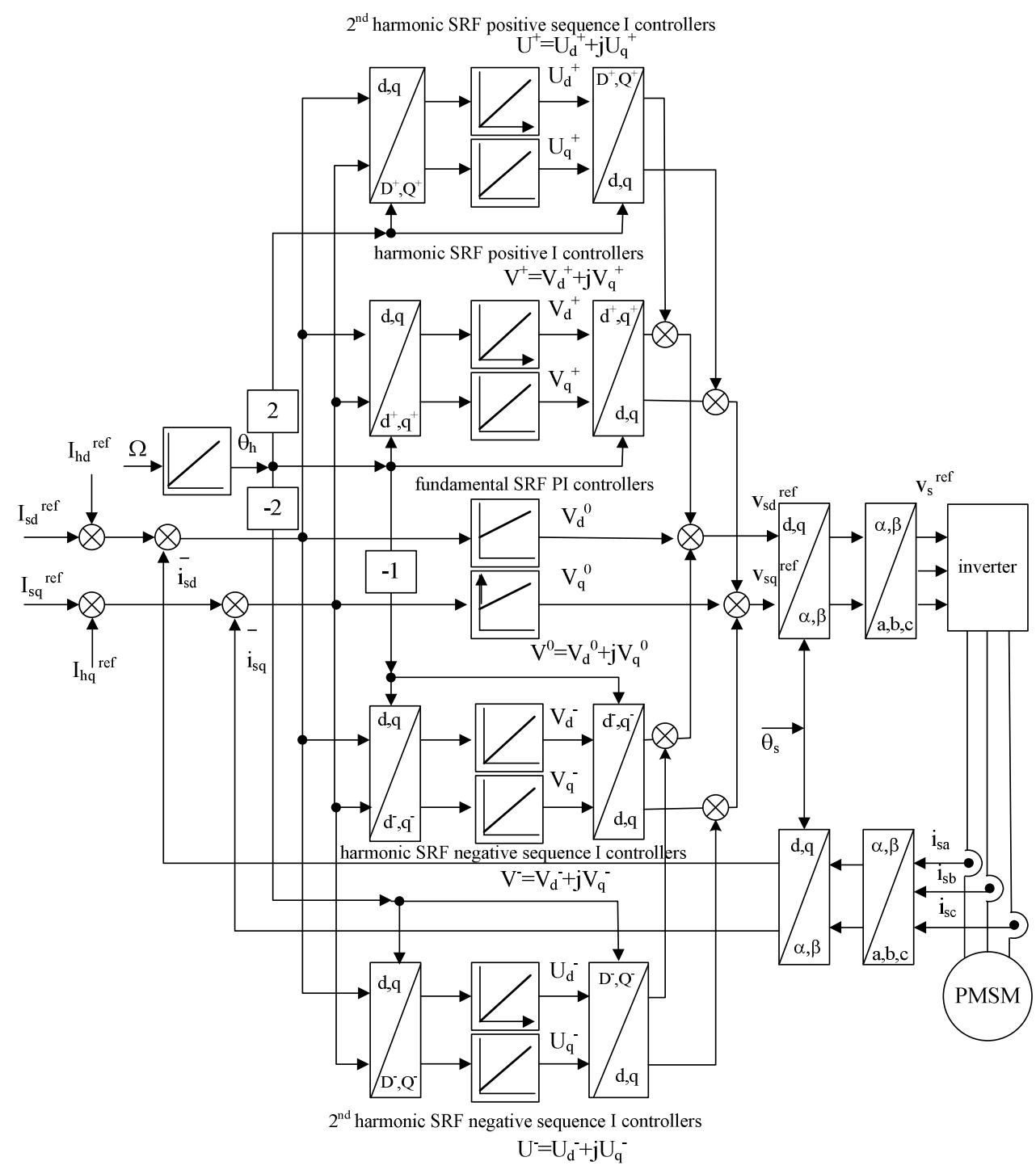

Fig. 2. Standard SRF fundamental frequency PI current controller augmented by harmonic SRF I controllers for harmonic current control and frequency/sequence decomposition of the stator voltage vector.

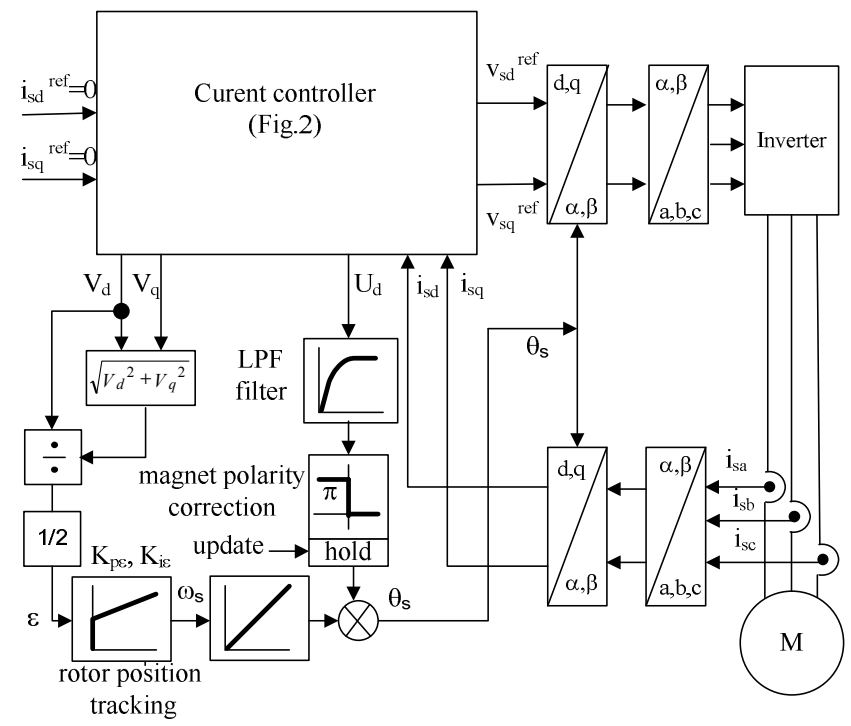

Fig. 3. Rotor position tracking loop. 


\section{ROTOR POSITION TRACKING}

As we have theoretically shown in Sections IV and V, the rotor flux position error signal can be retrieved directly from the current harmonic controller outputs $V_{d}^{-}$or $V_{d}$, depending on type of the injection.

After normalization the rotor position error is calculated:

$$
\varepsilon \approx \frac{1}{2} \frac{V_{d}}{\sqrt{V_{d}^{2}+V_{q}^{2}}}
$$

A PLL stile rotor flux position tracking loop driven by the error signal (23) can be introduced as shown in Fig. 3. The stator frequency is adjusted by a PI controller $\left(K_{p} \varepsilon\right.$ and $\left.K_{i \varepsilon}\right)$ until the position error signal $\varepsilon$ is reduced to zero:

$$
\omega_{s}(s)=-\left(K_{p \varepsilon}+\frac{K_{i \varepsilon}}{s}\right) \varepsilon(s)
$$

The position tracking loop ensures that the rotor position error will converge to zero and that the controller $\mathrm{d}$ axis is aligned with the rotor $\mathrm{d}$ axis. The $0-\pi$ uncertainty (ambiguity) about the rotor flux polarity is resolved in the manner discussed in Section VI.

\section{EXPERIMENTAL RESULTS}

The presented initial rotor position detection approach has been experimentally verified on a surface magnet PMSMs with low (saturation induced) saliency, $\mathrm{Ld}=90 \% \mathrm{Lq}$. The motor parameters are listed in Table I. In these tests the pulsating $\mathrm{d}$ axis harmonic current injection at $30 \mathrm{~Hz}$ was used with the current magnitude of $25 \%$ of the nominal current.

\section{TABLE I. MOTOR PARAMETERS}

\begin{tabular}{|l|l|}
\hline $\mathrm{I}_{\mathrm{n}}[\mathrm{A}]$ & $19.9 \mathrm{~A}$ \\
\hline $\mathrm{T}_{\mathrm{n}}[\mathrm{Nm}]$ & $25 \mathrm{Nm}$ \\
\hline $\mathrm{f}_{\mathrm{n}}$ & $200 \mathrm{~Hz}$ \\
\hline $\mathrm{J}\left[\mathrm{kgm}^{2}\right]$ & 0.00514 \\
\hline$n_{p}$ & 3 \\
\hline$\varphi_{\mathrm{m}}[\mathrm{Wb}$ peak $]$ & 0.2 \\
\hline $\mathrm{L}_{\mathrm{d}}[\mathrm{mH}]$ & 4.25 \\
\hline $\mathrm{L}_{\mathrm{q}}[\mathrm{mH}]$ & 4.75 \\
\hline
\end{tabular}

The controller parameterization and settings are listed in Table II. The fundamental current controller bandwidth was set to $50 \mathrm{~Hz}$ while the tracking loop bandwidth was set to $5 \mathrm{~Hz}$.

\section{TABLE II. CONTROLLER PARAMETERS}

\begin{tabular}{|c|c|}
\hline Parameter & Value \\
\hline $\mathrm{K}_{\mathrm{p}}$ & $\omega_{0} \mathrm{~L}$ \\
\hline $\mathrm{K}_{\mathrm{i}}$ & $\omega_{0}{ }^{2} \mathrm{~L}$ \\
\hline $\mathrm{K}_{\mathrm{ih}}$ & $\Omega^{2} \mathrm{~L}$ \\
\hline$\omega_{0}$ & $2 \pi \mathrm{f}_{0}$ \\
\hline $\mathrm{f}_{0}[\mathrm{~Hz}]$ & 50 \\
\hline $\mathrm{K}_{\mathrm{p} \varepsilon}$ & $2 \xi_{\varepsilon} \omega_{\varepsilon}$ \\
\hline $\mathrm{K}_{\mathrm{i} \varepsilon}$ & $\omega_{\varepsilon}{ }^{2}$ \\
\hline$\omega_{\varepsilon}$ & $2 \pi \mathrm{f}_{\varepsilon}$ \\
\hline $\mathrm{f}_{\varepsilon}[\mathrm{Hz}]$ & 5 \\
\hline$\xi_{\varepsilon}$ & 1 \\
\hline
\end{tabular}

Figure 4 shows that the rotor position tracking (based on regulating the normalised composite voltage $\mathrm{V}_{d}$ to zero) converges within $500 \mathrm{~ms}$. The rotor flux polarity is detected by observation of examining the sign of the second harmonic of the injected voltage at the end of the alignment phase. Figure 5 illustrates a situation when the controller position angle is corrected by $\pi$ after the tracking phase has been completed.

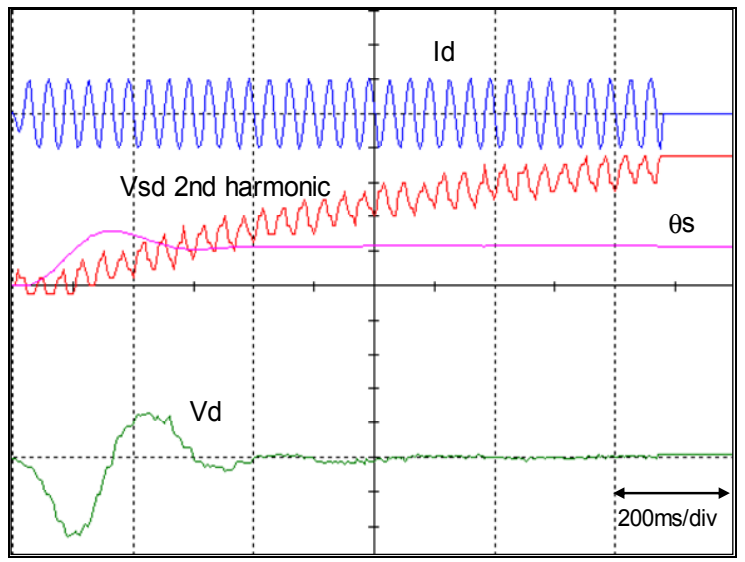

Fig. 4. Convergence of the position tracking in PMSM. Flux polarity detection based on observation of sign of second harmonic in the stator voltage reference (no correction by $\pi$ ).

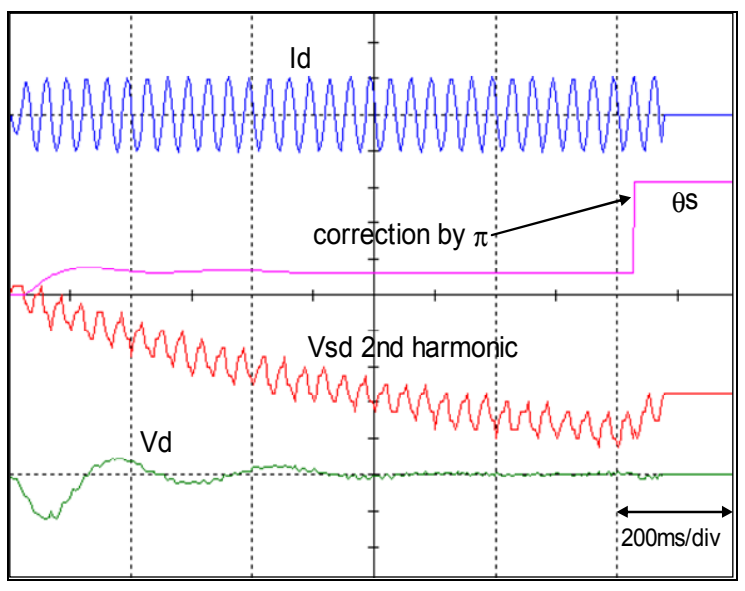

Fig. 5. Convergence of the position tracking in PMSM. Flux polarity detection based on observation of sign of second harmonic in the stator voltage reference (correction by $\pi$ ).

The detected initial rotor position angle was compared with the position sensor information in several tests with randomly selected rotor positions (Table III). The initial position accuracy was found to be well within \pm 15 electrical degrees.

TABLE III. COMPARISON OF THE DETECTED ROTOR POSITION WITH THE POSITION SENSOR INFORMATION

\begin{tabular}{|c|c|c|c|}
\hline Test & $\Delta \theta[$ deg.] & Test & $\Delta \theta[$ deg.] \\
\hline 1 & 3.4 & 6 & 1.5 \\
\hline 2 & -6.6 & 7 & -4.6 \\
\hline 3 & 11.3 & 8 & 6.5 \\
\hline 4 & 5.4 & 9 & -4.7 \\
\hline 5 & -6.6 & 10 & 7.3 \\
\hline
\end{tabular}


The initial rotor position detection method enabled smooth starts of the PMSM without uncontrolled movements. An advantage of this start-up method compared to various high frequency injection based methods is that it does not results in considerable noise and that it can be used with any type of PMSM rotor designs. However the tracking dynamics is relatively slow (several hundred of $\mathrm{ms}$ ).

\section{Conclusions}

This paper has presented an approach for initial rotor position detection in PMSM. It is based on a stator current controller which integrates two tasks: the stator current harmonic injection control with spectral decomposition of the stator voltage response. The current controller is based on a bank of SRF based integral controllers dedicated to every spectral component of interest. In this way the fundamental and harmonic current injections can be performed with zero steady-state error while respective spectral components of the stator voltage response are readily available at the SRF integrator outputs without any additional signal processing. By applying the perturbations techniques we have shown in a systematic manner links between the harmonic integral controller outputs and rotor position error in either induction or PMSM machines and with two characteristic types of harmonic injections (rotational and pulsating). Based on the theoretical analysis a rotor flux position tracking scheme has been constructed. Several experimental tests confirm the theoretical results and demonstrate that the method is applicable for the initial rotor position detection in PMSM.

Application of this rotor flux position tracking method can be further expended into sensorless control of PMSMs and induction machines in the low speed region [18]. However the tracking dynamics is relatively slow and inferior compared to that achievable by using various high frequency based injection techniques.

\section{REFERENCES}

[1] M.C. Harke, D. Raca, R.D. Lorenz, "Implementation Issues for Fast Initial Position and Magnet Polarity Identification of PM Synchronous Machines with Near Zero Saliency", European Conference on Power Electronics and Applications, EPE 2005, Dresden, pp. P.1-P.10.

[2] K. Tanaka; T. Yuzawa, R. Moriyama; I. Miki, "Initial Rotor Position Estimation for Surface Permanent Magnet Synchronous Motor", Conference Record of Thirty-Sixth IEEE IAS Annual Industry Applications Conference, 30 Sept.-4 Oct. 2001, Vol. 4, pp. $2592-2597$.

[3] J. Holtz, "Initial Rotor Polarity Detection and Sensorless Control of PM Synchronous Machines", Conference Record of the 41st IAS Annual Industry Applications Conference, 8-12 Oct. 2006, Vol. 4, pp. $2040-2047$.

[4] Y-S. Jeong, R. D. Lorenz, T.M. Jahns, S-K. Sul, "Initial Rotor Position Estimation of an Interior Peramanent-Magnet Synchronous Machine Using Carrier-Frequency Injection Methods“, IEEE Transactions on Industry Applications, Vol. 41, No. 1, Jan./Feb. 2005, pp. 38-45.

[5] D. Kaneko, Y. Iwaji, K. Sakamoto, T. Endo, "Initial Rotor Position Estimation of the Interior Permanent Magnet Synchronous Motor", Proceedings of the Power Conversion Conference, PCC Osaka, 2-5 April 2002, Vol. 1, pp. 259 - 264.

[6] M. Schroedl, "Sensorless control of AC machines at low speed and standstill based on the "INFORM" method", Conference
Record of the Thirty-First IEEE Industry Applications Society Annual Meeting, IAS '96, 6-10 Oct. 1996, Vol. 1, pp. 270-277.

[7] P.B. Schmidt; M.L. Gasperi; G. Ray; A.H. Wijenayake, "Initial Rotor Angle Detection of a Nonsalient Pole Permanent Magnet Synchronous Machine", Conference Record of the Thirty-Second IEEE IAS Annual Industry Applications Conference, IAS '97., 5-9 Oct. 1997, Vol. 1, pp. $459-463$.

[8] Joohn Sheok Kim, Seung Ki Sul, "New Stand-Still Position Detection Strategy for PMSM Drive without Rotational Transducers", Conference Proceedings of the Ninth Annual Applied Power Electronics Conference and Exposition, APEC '94., Vol. 1, 13-17 Feb. 1994, pp. 363 - 369.

[9] V-M. Leppanen, J. Luomi, "Rotor Flux Angle Tracking Controller for Sensorless Induction Motor Drives", IEEE Industry Applications Conference, $37^{\text {th }}$ IAS Annual Meeting, Vol. 2, Oct. 2002, pp. 856-863.

[10] V.-M. Leppanen, J. Luomi, "Speed-Sensorless Induction Machine Control for Zero Speed and Frequency", IEEE Transactions on Industrial Electronics, Vol. 51, No. 5, Oct. 2004, pp. 1041-1047.

[11] T. Kereszty, V-M Leppanen, J. Luomi, "Sensorless Control of Surface Magnet Synchronous Motors at Low Speeds Using LowFrequency Signal Injection”, IECON'03, Nov. 2003, pp. 12391243.

[12] S. Wu, Y. Li, X. Miao, "Comparison of Signal Injection Methods for Sensorless control of PMSM at Very Low Speeds", IEEE Power Electronics Specialists Conference, PESC 2007, June 2007, pp. $568-573$.

[13] M. Eskola, H. Tuusa, "Sensorless Control of Salient Pole PMSM Using a Low -Frequency Signal Injection", European Conference on Power Electronics and Applications, Sept. 2005, pp. 1-10.

[14] S.J. Lee, S.K. Sul, "A Harmonic Reference Frame Based Current Controller for Active Filter", Fifteenth Annual IEEE Applied Power Electronics Conference and Exposition, APEC 2000, Vol. 2, 6-10 Feb. 2000, pp. $1073-1078$.

[15] D. Basic, V.S. Ramsden, P.K. Muttik, "Harmonic Filtering of High-Power 12-Pulse Rectifier Loads with a Selective Hybrid Filter System", IEEE Transactions on Industrial Electronics, Vol. 48, No. 6, Dec. 2001, pp. 1118 - 1127.

[16] Hassan K. Khalil, "Nonlinear Systems", Prentice Hall, Third Edition, 2002, Chapter 10.4, Pages 402-411.

[17] M. Hinkkanen, V-M. Leppanen, J. Luomi, "Flux Observer Enhanced with Low-Frequency Signal Injection Allowing Sensorless Zero-Frequency Operation of Induction Motors“, IEEE Transactions on Industry Applications, Vol. 41, No. 1, Jan./Feb. 2005, pp. 52-59.

[18] D. Basic, F. Malarit, P. Rouchon, "Current Controller for Low Frequency Harmonic Injection and Rotor Flux Position Tracking at Low Speeds", 2010, Unpublished Paper. 